\title{
Covariant Derivatives on Null Submanifolds
}

\author{
Don Hickethier \\ Department of Mathematics, Flathead Valley Community College, Kalispell, MT 59901 \\ dhicketh@fvcc.edu \\ Tevian Dray \\ Department of Mathematics, Oregon State University, Corvallis, OR 97331 \\ tevian@math.oregonstate.edu
}

July 26, 2011

\begin{abstract}
The degenerate nature of the metric on null hypersurfaces makes it difficult to define a covariant derivative on null submanifolds. Recent approaches using decomposition to define a covariant derivative on null hypersurfaces are investigated, with examples demonstrating the limitations of the methods. Motivated by Geroch's work on asymptotically flat spacetimes, conformal transformations are used to construct a covariant derivative on null hypersurfaces, and a condition on the Ricci tensor is given to determine when this construction can be used. Several examples are given, including the construction of a covariant derivative operator for the class of spherically symmetric hypersurfaces.
\end{abstract}

\section{Introduction}

Given a null submanifold of a Lorentzian spacetime, is it possible to define a preferred torsion-free, metric-compatible covariant derivative? Is it possible to determine, a priori, when such a connection can be found?

One place where the need for a such a derivative arises is in the study of asymptotically flat spacetimes. According to Geroch [2]:

In the null case, one has no unique derivative operator, and so one works more with Lie and exterior derivatives, and with other differential concomitants. As a general rule, it is considerably more difficult in the null case to write down formulae which say what one wants to say.

Geroch's treatment of the null boundaries of asymptotically flat spacetimes [2] motivates the techniques used here to find a preferred derivative operator on (some) null submanifolds. 
We briefly review traditional approaches to this problem in Section 2, and summarize the relevant parts of Geroch's construction of a null asymptotic boundary in Section 3 , Section 4 establishes an existence condition, showing when the Geroch construction can be used to construct a preferred covariant derivative on a given null submanifold, and Section 5 then gives a simpler condition on the Ricci tensor for determining when this construction is possible. We present several examples in Section 6, including the horizon of the Schwarzschild geometry, and discuss our results in Section 7.

\section{Traditional Approaches}

\subsection{Gauss decomposition}

Let $(M, g)$ be a spacetime, that is a manofild $M$ together with a nondegenerate metric $g$ of Lorentzian signature. If $(\Sigma, q)$ is a submanifold of $(M, g)$ given by $\varphi: \Sigma \rightarrow M$, and if $q=\varphi^{*} g$ is a nondegenerate metric on $\Sigma$, then a connection $\nabla$ on $M$ induces a natural connection $D$ on $\Sigma$. A traditional approach to defining this connection is to split $T M$ into the direct sum

$$
T M=T \Sigma \oplus T \Sigma^{\perp},
$$

where $T \Sigma^{\perp}$ is the orthogonal complement of $T \Sigma$ in $T M$.

For $X, Y \in \Gamma(T M), \nabla_{X} Y$ can be separated on $\Sigma$ into tangential and orthogonal components of $T M$ which define the induced connection, $D_{X} Y$, and the second fundamental form, $I I(X, Y)$. Explicitly, we have

$$
\begin{aligned}
D_{X} Y & =\left(\nabla_{X} Y\right)^{\|} \\
I I(X, Y) & =\left(\nabla_{X} Y\right)^{\perp}=\nabla_{X} Y-D_{X} Y .
\end{aligned}
$$

If $\nabla$ is the Levi-Civita connection on $M$, then $D$ turns out to be the Levi-Civita connection on $\Sigma$. The decomposition

$$
\nabla_{X} Y=D_{X} Y+I I(X, Y)
$$

is called Gauss' formula [4].

\subsection{Duggal decomposition}

Difficulties arise when the metric $q$, on $\Sigma$, is degenerate. Furthermore, if $\Sigma$ is lightlike, $T M$ cannot be decomposed into the direct sum of $T \Sigma$ and $T \Sigma^{\perp}$, since there are vectors in $T \Sigma$ that are also in $T \Sigma^{\perp}$, as well as vectors that are in neither space. Despite these difficulties, Duggal and Bejancu [3] (henceforth referred to as Duggal) introduced a decomposition that produces equations similar to the Gauss formula (4), as we now describe.

Given a lightlike submanifold $\Sigma$ of $M$ with tangent space $T \Sigma$, the goal is to create a decomposition of $T M$ by producing a vector bundle similar to $T \Sigma^{\perp}$. Choose a screen manifold $\operatorname{Ser}(T \Sigma) \subset T \Sigma$ such that

$$
T \Sigma=\operatorname{Scr}(T \Sigma) \oplus T \Sigma^{\perp}
$$


Given a screen manifold, $\operatorname{Scr}(T \Sigma)$, Duggal proves the existence of a unique complementary vector bundle, $\operatorname{tr}(T \Sigma)$, to $T \Sigma$, called the lightlike transversal vector bundle of $\Sigma$ with respect to $\operatorname{Scr}(T \Sigma)$.

Theorem 1 (Duggal). Let $(\Sigma, q, S c r(T \Sigma))$ be a lightlike hypersurface of a Lorentzian manifold $(M, g)$. Then there exists a unique vector bundle $\operatorname{tr}(T \Sigma) \subset T M$, of rank 1 over $\Sigma$, such that for any nonzero $\xi \in \Gamma\left(T \Sigma^{\perp}\right)$ there exists a unique $N \in \Gamma(\operatorname{tr}(T \Sigma))$ such that

$$
N \cdot \xi=-1, \quad N \cdot N=0, \quad N \cdot W=0 \forall W \in \operatorname{Scr}(T \Sigma) .
$$

Thus, $\operatorname{tr}(T \Sigma) \perp S c r(T \Sigma)$ and since $\operatorname{tr}(T \Sigma)$ is 1 -dimensional, $\Gamma(\operatorname{tr}(T \Sigma))=\operatorname{Span}(N)$. By construction we have $\operatorname{tr}(T \Sigma) \cap T \Sigma=\{0\}$ and have decomposed $T M$ to

$$
T M=\operatorname{Scr}(T \Sigma) \oplus\left(T \Sigma^{\perp} \oplus \operatorname{tr}(T \Sigma)\right)=T \Sigma \oplus \operatorname{tr}(T \Sigma)
$$

where $T M$ is restricted to $\Sigma$.

We can use (7) to decompose the connection $\nabla$ on $M$ as follows. Let $X, Y \in \Gamma(T \Sigma)$ and $V \in \Gamma(\operatorname{tr}(\Sigma))$. Then, since $\operatorname{tr}(T \Sigma)$ has $\operatorname{rank} 1$, we can write

$$
\begin{aligned}
& \nabla_{X} Y=D_{X} Y+B(X, Y) N \\
& \nabla_{X} V=-A_{N} X+\tau(X) N,
\end{aligned}
$$

where $D_{X} Y, A_{N} X \in \Gamma(T \Sigma)$. Equation (8) can be thought of as the Gauss formula for the lightlike hypersurface and (9) as the lightlike Weingarten formula. Under this decomposition, $D_{X} Y$ is a connection on $\Sigma$, but, as discussed in Duggal [3], this connection is not, in general, metric-compatible.

\subsection{Example}

To investigate the Duggal decomposition, consider the line element

$$
d s^{2}=-2 d u d v+q_{i j} d x^{i} d x^{j}
$$

Choose a screen $\operatorname{Scr}(T \Sigma)=\operatorname{Span}\left(\left\{X_{1}, X_{2}\right\}\right)$ by setting

$$
X_{k}=\frac{\partial}{\partial x^{k}}+\alpha_{k} \xi
$$

where $\xi=\eta \frac{\partial}{\partial v} \in \Gamma\left(T \Sigma^{\perp}\right)$ and $\alpha_{k}$ is a function of $v, x^{1}$ and $x^{2}$. All possible screens can be obtained by choosing different $\alpha_{k}$. For $N \in \Gamma(\operatorname{tr}(T \Sigma))$ satisfying Theorem 1, the vectors $\left\{\xi, X_{1}, X_{2}, N\right\}$ form a basis for $\Gamma(T M)$. As shown in Duggal [3], the covariant derivatives take the form

$$
\begin{aligned}
\nabla_{X_{j}} X_{i} & =\gamma^{0}{ }_{i j} \xi+\gamma^{k}{ }_{i j} X_{k}+B_{i j} N \\
\nabla_{X_{j}} \xi & =\gamma^{0}{ }_{0 j} \xi+\gamma^{k}{ }_{0 j} X_{k} \\
\nabla_{\xi} X_{i} & =\gamma^{0}{ }_{i 0} \xi+\gamma^{k}{ }_{i 0} X_{k} \\
\nabla_{\xi} \xi & =\gamma^{0}{ }_{00} \xi
\end{aligned}
$$


and

$$
\begin{aligned}
\nabla_{X_{j}} N & =-A^{k}{ }_{j} X_{k}+\tau_{j} N \\
\nabla_{\xi} N & =-A^{k}{ }_{0} X_{k}+\tau_{0} N
\end{aligned}
$$

Duggal defines the last term in equation (12a) to be the second fundamental form,

$$
I I(X, Y)=B(X, Y) N
$$

Thus, equations (12a) $-(12 \mathrm{~d})$ decompose $\nabla$ to a form similar to Gauss' formula (8) . Once the coefficients in (12a) - (12d) are known, a connection $D$ on $\Sigma$ has been constructed. The properties of the Levi-Civita connection $\nabla$ on $\Sigma$ can be used to show that

$$
\begin{aligned}
\gamma^{k}{ }_{i j} & =\frac{1}{2} q^{k h}\left(X_{j}\left(q_{i h}\right)+X_{i}\left(q_{h j}\right)-X_{h}\left(q_{i j}\right)\right) \\
\gamma^{0}{ }_{i j} & =-g_{i k} A^{k}{ }_{j} \\
\gamma^{0}{ }_{0 j} & =-\tau_{j} \\
\gamma^{k}{ }_{0 j} & =\frac{1}{2} g^{i k} \xi\left(g_{i j}\right) \\
\gamma_{00}^{0} & =-\tau_{0} \\
B_{i j} & =\frac{1}{2} \xi\left(g_{i j}\right) .
\end{aligned}
$$

and the induced covariant derivative on $T \Sigma$ becomes

$$
\begin{aligned}
D_{X_{j}} X_{i} & =\gamma^{0}{ }_{i j} \xi+\gamma^{k}{ }_{i j} X_{k} \\
D_{X_{j}} \xi & =\gamma^{0}{ }_{0 j} \xi+\gamma^{k}{ }_{0 j} X_{k} \\
D_{\xi} X_{i} & =\gamma^{0}{ }_{i 0} \xi+\gamma^{k}{ }_{i 0} X_{k} \\
D_{\xi} \xi & =\gamma^{0}{ }_{00} \xi
\end{aligned}
$$

\subsection{Uniqueness}

In general, the above construction of the induced covariant derivative $D$ depends on the choice of screen, that is, on the choice of $X_{k}$. However, Duggal further proves that under certain conditions there is a unique induced connection on $\Sigma$.

Theorem 2 (Duggal). Let $(\Sigma, q, \operatorname{Scr}(T \Sigma))$ be a lightlike hypersurface of $(M, g)$. Then the induced connection $D$ is unique, that is, $D$ is independent of $\operatorname{Scr}(T \Sigma)$, if and only if the second fundamental form II vanishes identically on $\Sigma$. Furthermore, in this case, $D$ is torsion free and metric compatible.

Theorem 2 implies that if $B_{i j} \neq 0$, or equivalently, $\xi\left(g_{i j}\right) \neq 0$ for all $i, j$, then there is a need for a new method to define a covariant derivative on $\Sigma$. 
Returning to our example (10), if the 2-metric $q_{i j}$ is that of a plane, that is, if we consider a null plane $\Sigma=\{u=0\}$ in Minkowski space $\mathbb{M}^{4}$ in null rectangular coordinates with line element

$$
d s^{2}=-2 d u d v+d x^{2}+d y^{2}
$$

then it is straightforward to show that $B_{i j}=0$, so that we obtain a unique connection on $\Sigma$ regardless of the screen chosen. It is a useful exercise to check this explicitly, using

$$
X_{1}=\alpha \frac{\partial}{\partial v}+\frac{\partial}{\partial x}, \quad X_{2}=\beta \frac{\partial}{\partial v}+\frac{\partial}{\partial y}, \quad \xi=\eta \frac{\partial}{\partial v}
$$

with arbitrary $\alpha, \beta, \eta$, which implies

$$
N=\frac{1}{\eta}\left(\frac{\partial}{\partial u}+\left(\frac{\alpha^{2}+\beta^{2}}{2}\right) \frac{\partial}{\partial v}+\alpha \frac{\partial}{\partial x}+\beta \frac{\partial}{\partial y}\right)
$$

However, if the 2-metric $q_{i j}$ is that of a sphere, that is, if we consider a null cone $\Sigma=$ $\{u=0\}$ in Minkowski space $\mathbb{M}^{4}$ in null spherical coordinates with line element

$$
d s^{2}=-2 d u d v+r^{2} d \theta^{2}+r^{2} \sin ^{2} \theta d \phi^{2}
$$

with $r=(v-u) / \sqrt{2}$, then $B_{i j} \neq 0$, since $q_{i j}$ depends on $r$. Thus, Duggal's construction using the screen distribution and transversal vector bundle does not yield a preferred, metriccompatible, torsion-free connection on the null cone.

An alternate construction will be developed in this paper.

\subsection{Connections via the Pullback}

Another possible way to construct a connection on a submanifold is to use pullbacks. Although this method often fails, we will show in subsequent sections that a modified version of this method has wide applicability.

Let $\Sigma$ be a submanifold of $\mathbb{M}^{4}$ with $\varphi: \Sigma \rightarrow \mathbb{M}^{4}$ an embedding of $\Sigma$ into $\mathbb{M}^{4}$. If $w_{a}$ is a 1 -form on $\mathbb{M}^{4}$, its pullback $\varphi^{*} w_{a}$ is a 1 -form on $\Sigma$. We can therefore attempt to define a covariant derivative $D$ on $\Sigma$ by pulling back the covariant derivative operator $\nabla$ on $\mathbb{M}^{4}$, that is, we seek an operator $D$ satisfying

$$
D_{a}\left(\varphi^{*} w_{b}\right)=\varphi^{*}\left(\nabla_{a} w_{b}\right)
$$

We adopt a less formal notation, and write $w_{b}$ instead of $\varphi^{*} w_{b}$ for the pullback of $w_{b}$ to $\Sigma$. With this new notation, the pullback of the covariant derivative is written

$$
D_{a} \underset{\leftarrow}{w_{b}}=\underset{\nabla_{a} w_{b}}{\longleftarrow}
$$

It is easily checked that the Duggal connection on a null plane in Minkowski space, as defined in Section 2.2 and shown to be unique in Section 2.4, also satisfies (22), and is in 
fact uniquely defined by this condition. In general, however, (22) alone is not enough to determine a well-defined covariant derivative $D$ on $\Sigma$.

The problem is that there are many 1 -forms $w$ with the same pullback $w$; for $D$ to be well-defined on $\Sigma$, it must not depend on this choice. If $\Sigma=\{u=0\}$, then

$$
\stackrel{w+f d u}{\longleftarrow}=\stackrel{w}{\longleftarrow}
$$

since $d u=0$. Thus, (22) will be well-defined if (and only if)

$$
\stackrel{\nabla_{X} d u}{\longleftarrow}=0
$$

for all $X \in \Gamma(T \Sigma)$.

As an example, consider the null cone in Minkowski space given by $u=t-r=0$. Then

$$
\underset{\nabla_{X} d u}{\longleftarrow}=\underset{\nabla_{X} d t}{\longleftarrow}-\underset{\nabla_{X} d r}{\longleftarrow} \neq 0
$$

since $\underset{\nabla_{X} d t}{\nabla}=0$ but $\underset{\nabla_{X} d r}{\nabla} \neq 0$. Thus, the pullback method fails on the null cone.

In terms of coordinates $\left\{x^{i}\right\}$ on the surface $\Sigma=\{u=0\}$, extended to a neighborhood of $\Sigma$, it is easily seen that condition (22) for the existence of a well-defined pullback connection is equivalent to the vanishing of the appropriate Christoffel symbols, namely

$$
\Gamma_{i j}^{u}=0
$$

In the case of the null cone, we have

$$
\Gamma_{\theta \theta}^{u}=-\frac{v-u}{2}=-r=\frac{1}{\sin ^{2} \theta} \Gamma_{\phi \phi}^{u}
$$

The simple dependence of these terms on $r$ suggests a possible strategy: remove the $r$ dependence by rescaling the line element by $r^{2}$, after which these Christoffel symbols will vanish, and a well-defined covariant derivative can be defined.

We implement this strategy in the remainder of the paper

\section{Asymptotically Flat Spacetimes}

There is a well-known context in general relativity for studying a null submanifold, namely the construction of null infinity for an asymptotically flat spacetime. Since much of that construction will be useful in our more general context, we briefly review it here. Our presentation follows the classic 1976 paper of Geroch [2].

An asymptote of a spacetime $\left(\widetilde{M}, \widetilde{g}_{a b}\right)$ is a manifold $M$ with boundary $\mathscr{I}$, together with a smooth Lorentzian metric $g_{a b}$ on $M$, a smooth function $\Omega$ on $M$, and a diffeomorphism by means of which we identify $\bar{M}$ and $M-\mathscr{I}$, satisfying the following conditions:

1. On $\widetilde{M}, g_{a b}=\Omega^{2} \widetilde{g}_{a b}$;

2. On $\mathscr{I}, \Omega=0, \nabla_{a} \Omega \neq 0$, and $g^{a b}\left(\nabla_{a} \Omega\right)\left(\nabla_{b} \Omega\right)=0$, where $\nabla_{a}$ denotes covariant differentiation on $M$. 
The Ricci curvature tensors of the conformally related metrics $g_{a b}$ and $\widetilde{g}_{a b}$ are related by

$$
\begin{aligned}
\widetilde{R}_{a b}=R_{a b} & +(s-2) \Omega^{-1} \nabla_{a} \nabla_{b} \Omega+\Omega^{-1} g_{a b} \nabla^{m} \nabla_{m} \Omega \\
& -(s-1) \Omega^{-2} g_{a b}\left(\nabla^{m} \Omega\right)\left(\nabla_{m} \Omega\right)
\end{aligned}
$$

where $s$ is the dimension of $M$.

We introduce the normal vector field

$$
n^{a}=g^{a k} \nabla_{k} \Omega=\nabla^{a} \Omega
$$

and compute

$$
£_{n} g_{a b}=2 \nabla_{a} \nabla_{b} \Omega
$$

Following Geroch [2], we assume that the physical stress-energy tensor vanishes asymptotically to order 2 , that is, we assume that $\Omega^{-2} \widetilde{R}_{b}{ }_{b}$ admits a smooth extension to $\mathscr{I}$, which in turn implies that $\Omega \widetilde{R}_{a b}$ is zero on $\mathscr{I}$. 1 As shown by Geroch [2], we can then use the gauge freedom in the choice of $\Omega$ to ensure that the pullback to $\mathscr{I}$ of the RHS of (30) vanishes. Explicitly, by solving the ordinary differential equation

$$
n^{c} \nabla_{c} \ln \omega=-\frac{1}{s} \square \Omega
$$

along each integral curve of $n^{a}$ on $\mathscr{I}$, where $\square \Omega=g^{a b} \nabla_{a} \nabla_{b} \Omega$ is the d'Alembertian, and setting $\bar{\Omega}=\omega \Omega$, so that

$$
\begin{aligned}
\bar{g}_{a b} & =\omega^{2} g_{a b} \\
\bar{n}^{a} & =\omega^{-1} n^{a}
\end{aligned}
$$

then

$$
\stackrel{£_{\bar{n}} \bar{g}_{a b}}{\longleftarrow}=0
$$

Thus, given an asymptotically flat spacetime satisfying the original Geroch conditions, one can assume without loss of generality that $n$ is in fact a Killing vector field.

A divergence-free conformal frame $\left(g_{a b}, n^{c}\right)$ satisfying (34) (where we have dropped the bars) has an additional property: the pullback connection is well-defined on the null submanifold $\mathscr{I}$. To see this, we set

$$
q_{a b}=g_{a b}
$$

and note that

$$
£_{n} q_{a b}=£_{n} g_{a b}=2_{\longleftarrow}^{2} \nabla_{a} n_{b} .
$$

Setting $u=\Omega$, the 1 -form $n_{b}$ is just $d u$, so that

$$
\left(£_{n} g\right)_{i j}=-2 \Gamma^{u}{ }_{i j} .
$$

and the result now follows by comparison with (26)).

\footnotetext{
${ }^{1}$ We will weaken this assumption below, which will affect the numerical factor in (31).
} 


\section{Covariant Derivatives on Null Submanifolds}

We now adapt the results from the previous section for asymptotically flat spacetimes to more general null submanifolds.

Recall that a vector field $v^{a}$ is Killing if $£_{v} g_{a b}=0$. Given a null surface, our first result is that if the normal vector is Killing, then there is a well-defined covariant derivative on $\Sigma$. Following Geroch, we then consider the conditions under which a conformally related metrics admits a well-defined covariant derivative. When appropriate conditions are satisfied, we further propose that the resulting notion of covariant derivative be regarded as the natural choice on the null submanifold.

Lemma 1 (Covariant Derivative on $\Sigma$ ). Let $\Sigma=\{u=0\}$ be a null submanifold of a given spacetime $\left(M, g_{a b}\right)$, let $n_{a}=\nabla_{a} u$, and let $q_{a b}$ be the induced degenerate metric on $\Sigma$, as in (35). If $£_{n} q_{a b}=0$ on $\Sigma$, then the connection defined by the pullback, as in (22), is well-defined.

Proof. Let $w_{b}$ be any 1 -form on the surface $\Sigma$. We would like to define a covariant derivative using the pullback, $D_{a} w_{b}=\nabla_{a} W_{b}$, where $W_{b}$ is a 1 -form on $M$ such that $\underset{W_{b}}{\longleftarrow}=w_{b}$, but we must show that this is well defined.

On $\Sigma, n_{b}=0$, since $n_{b} d x^{b}=d u$. Let $V_{b}=W_{b}+k n_{b}$ where $k$ is any function. $V_{b}$ is the most general 1-form with the same pullback as $W_{b}$,

$$
\underset{V_{b}}{\leftarrow}=\underset{W_{b}}{\longleftarrow}+\underset{\leftarrow}{k} n_{b}=w_{b}+0
$$

Consider the pullback of the derivative of $V_{b}-W_{b}$,

$$
\begin{aligned}
& \underset{\nabla_{a}\left(V_{b}-W_{b}\right)}{\longleftarrow}=\underset{ }{\nabla_{a}\left(k n_{b}\right)} \\
& =\left(\underset{\longleftarrow}{\nabla_{a} k}\right) n_{\leftarrow}+\left.k\right|_{u=0}\left(\underset{\longleftarrow}{\nabla_{a} n_{b}}\right) \\
& =\left.\frac{1}{2} k\right|_{u=0}\left(£_{n} q_{a b}\right)=0
\end{aligned}
$$

by assumption, where we have used (36) in the penultimate equality.

Lemma 2 (Conformal Killing Vector). With $\Sigma, n^{a}$, and $q_{a b}$ as above, if $£_{n} q_{a b}=f q_{a b}$, then there exists a unique conformal factor $\omega$, up to a constant factor, such that $£_{\bar{n}} \bar{q}_{a b}=0$, with $\bar{n}^{a}$ and $\bar{q}_{a b}$ as in (33).

Proof. This is essentially the same as the result in Geroch [2] quoted above, and the proof is similar. We have

$$
\begin{aligned}
£_{\bar{n}} \overline{g_{a b}} & =\bar{n}^{c} \nabla_{c} \bar{g}_{a b}+\bar{g}_{c b} \nabla_{a} \bar{n}^{c}+\bar{g}_{a c} \nabla_{b} \bar{n}^{c} \\
& =\omega^{-1} n^{c} \nabla_{c}\left(\omega^{2} g_{a b}\right)+\omega^{2} g_{c b} \nabla_{a}\left(\omega^{-1} n^{c}\right)+\omega^{2} g_{a c} \nabla_{b}\left(\omega^{-1} n^{c}\right) \\
& =n^{c}\left(2\left(\nabla_{c} \omega\right) g_{a b}-g_{c b} \nabla_{a} \omega-g_{a c} \nabla_{b} \omega\right)+\omega £_{n} g_{a b} .
\end{aligned}
$$


and pulling both sides back to $\Sigma$ results in

$$
2\left(n^{c} \nabla_{c} \omega\right) q_{a b}=-\omega f q_{a b} .
$$

Letting $\dot{\omega}=n^{c} \nabla_{c} \omega$ the equation simplifies to

$$
\frac{\dot{\omega}}{\omega}=-\frac{f}{2}
$$

This ordinary differential equation will have a unique solution, up to a constant factor, along each integral curve of $n^{a}$, yielding an $\omega$ such that the conformal transformation will result in $£ \bar{n} \overline{q_{a b}}=0$.

These two lemmas immediately yield the following result:

Theorem 3 (Covariant Derivative with conformal transformation). With $\Sigma, n^{a}$, and $q_{a b}$ as above, if $£_{n} q_{a b}=f q_{a b}$ on $\Sigma$, then the conformal pullback method produces a well-defined covariant derivative, $D$, on $\Sigma$.

Proof. Since $£_{n} q_{a b}=f q_{a b}$, Lemma 2 gives an $\omega$ such that under the conformal transformation $£_{\bar{n}} \overline{q_{a b}}=0$. Now by Lemma 1 , define the covariant derivative by $D=\underline{\nabla}$.

It is straightforward to verify that all of the examples considered so far satisfy the conditions in Theorem 3. The most interesting case is the null cone (20), for which

$$
£_{n} q_{a b}=\frac{2}{v} q_{a b}
$$

Substituting $f=2 / v$ into (42) gives the ordinary differential equation

$$
\frac{\dot{\omega}}{\omega}=\frac{1}{\omega} \frac{\partial \omega}{\partial v}=-\frac{1}{v}
$$

with solution

$$
\omega=\frac{c}{v}
$$

where $c$ is a constant. But

$$
\left.\frac{1}{r}\right|_{u=0}=\left.\frac{\sqrt{2}}{v-u}\right|_{u=0}=\frac{\sqrt{2}}{v}
$$

and we see that rescaling the line element by $1 / r^{2}$ leads to a well-defined covariant derivative, as previously conjectured.

It is worth noting that if we regard the null cone as the unphysical space in Geroch's construction, then the corresponding "physical" stress-energy tensor only vanishes asymptotically to order 1 , so that the derivation of (31) fails, although a similar result still holds, with a different constant of proportionality. We show in the next section that a weaker condition on the stress-energy tensor is indeed sufficient for the argument used here to work. 


\section{$5 \quad$ Ricci Tensor}

The techniques adapted from Geroch's work on asymptotically flat spacetimes have addressed the fundamental question: What are the conditions on a null surface needed to construct a well-defined covariant derivative? Either a Killing normal vector, $£_{n} q_{a b}=0$, or a conformal Killing vector, $£_{n} q_{a b}=f q_{a b}$, combined with a conformal transformation leads to a welldefined covariant derivative on a null surface $\Sigma$ using the pullback method.

One of the drawbacks of this construction is that the Lie derivative of the metric must first be computed on $M$ and then pulled back to $\Sigma$ to test the hypotheses of the theorems. If the hypotheses are met, we return to $M$, perform a conformal transformation if needed, compute $\nabla$, then pull this derivative back to $\Sigma$, giving $D$. It would be nice if there was a test to tell if the pullback led to a well-defined covariant derivative on $\Sigma$ and if a conformal transformation is required before pulling $\nabla$ back to $\Sigma$. Again the work of Geroch leads to precisely such a condition

Theorem 4 (Ricci Tensor and Covariant Derivative on $\Sigma$ ). Given a spacetime $\left(M, g_{a b}\right)$ containing a null surface $\Sigma=\{u=0\}$, then if $\Omega\left(R_{a b}-\widetilde{R}_{a b}\right)=k q_{a b}$, where $\widetilde{g}_{a b}=\Omega^{-2} g_{a b}$, $\Omega=u$, and where $R_{a b}$ and $\widetilde{R}_{a b}$ are the Ricci tensors of $g_{a b}$ and $\widetilde{g}_{a b}$ respectively, then the conformal pullback method leads to a well-defined connection on $\Sigma$.

Proof. From (28) and (30),

$$
\Omega \widetilde{R}_{a b}=\Omega R_{a b}+\frac{s-2}{2} £_{n} g_{a b}+g_{a b} \nabla^{m} \nabla_{m} \Omega-\frac{s-1}{\Omega} g_{a b}\left(\nabla^{m} \Omega\right)\left(\nabla_{m} \Omega\right) .
$$

Taking the trace of (28) (using the metric $g$ ) yields

$$
\frac{1}{\Omega}\left(\nabla^{m} \Omega\right)\left(\nabla_{m} \Omega\right)=\frac{2}{s} \nabla^{m} \nabla_{m} \Omega+\frac{1}{s(s-1)}\left(\Omega R-\Omega^{-1} \widetilde{R}\right)
$$

thus showing that the LHS admits a smooth limit to $\Sigma$ (since the last term on the RHS does by assumption). Setting $\Omega=u$ in (47) and using (48) and our hypotheses yields

$$
£_{n} q_{a b}=\underbrace{£_{a b}}_{n}=\left.\left(-\frac{2 k}{s-2}+\frac{2}{s} \nabla^{m} \nabla_{m} \Omega+\frac{2}{s(s-2)} \Omega^{-1} \widetilde{R}\right)\right|_{u=0} q_{a b}
$$

Theorem 3 now implies that there is a covariant derivative $D$ on $\Sigma$.

While the above derivation can be found in Geroch [2, the interpretation is quite different. We start with a metric $g_{a b}$, a null surface $\Sigma=\{u=0\}$ and a conformal factor defined by $\Omega=u$. In the sense of Geroch, we are creating an artificial "physical" space $\left(\widetilde{M}, \widetilde{g}_{a b}\right)$ in order to determine if the pullback method will result in a well-defined covariant derivative. In the Geroch approach, the boundary at null infinity was separated from $\widetilde{M}$ by the conformal transformation in order to define some structure of the null surface. In our approach, one begins with the null surface and uses the "physical" space to define the covariant derivative. 


\section{$6 \quad$ Further Examples}

The Schwarzschild metric in double-null Kruskal-Szekeres coordinates is given by

$$
d s^{2}=-\frac{32 m^{3}}{r} e^{-r / 2 m} d u d v+r^{2} d \theta^{2}+r^{2} \sin ^{2} \theta d \phi^{2}
$$

where $r$ is given implicitly by

$$
u v=\left(1-\frac{r}{2 m}\right) e^{r / 2 m}
$$

The Schwarzschild metric is a vacuum solution of Einstein's equation, so the Ricci tensor vanishes. Considering the horizon at $u=0$ and using (28) with $\Omega=u$, the relevant components of the conformally related Ricci tensor are given by

$$
\begin{aligned}
\widetilde{R}_{v v} & =0 \\
\widetilde{R}_{\theta \theta} & =\frac{r}{m} \\
\widetilde{R}_{\phi \phi} & =\frac{r}{m} \sin ^{2} \theta
\end{aligned}
$$

and we have

$$
\stackrel{\Omega\left(R_{a b}-\widetilde{R}_{a b}\right)}{\longleftarrow}=\underline{u}\left(R_{a b}-\widetilde{R}_{a b}\right)=0,
$$

trivially satisfying the necessary conditions of Theorem 4. Straightforward computation verifies that the pullback connection constructed from the conformally related metric

$$
\widetilde{d s}^{2}=-\frac{32 m^{3}}{r^{3}} e^{-r / 2 m} d u d v+d \theta^{2}+\sin ^{2} \theta d \phi^{2} .
$$

is well-defined, and can therefore be used on the horizon.

More generally, a spherically symmetric space times has a line element of the form

$$
d s^{2}=h d u d v+r^{2} d \theta^{2}+r^{2} \sin ^{2} \theta d \phi^{2}
$$

where $h$ and $r$ are both functions of the null coordinates $u$ and $v$. As in previous examples, the null surface is chosen to be $\Sigma=\{u=0\}$. The nonzero components of the Ricci tensor are

$$
\begin{aligned}
R_{u u} & =-\frac{2\left(h \frac{\partial^{2} r}{\partial u^{2}}-\frac{\partial h}{\partial u} \frac{\partial r}{\partial u}\right)}{h r} \\
R_{u v} & =\frac{r \frac{\partial h}{\partial u} \frac{\partial h}{\partial v}-h r \frac{\partial^{2} h}{\partial v \partial u}-2 h^{2} \frac{\partial^{2} r}{\partial v \partial u}}{h^{2} r} \\
R_{v v} & =-\frac{2\left(h \frac{\partial^{2} r}{\partial v^{2}}-\frac{\partial h}{\partial v} \frac{\partial r}{\partial v}\right)}{h r} \\
R_{\theta \theta} & =-\frac{4 r \frac{\partial^{2} r}{\partial v \partial u}-h+4 \frac{\partial r}{\partial v} \frac{\partial r}{\partial u}}{h} \\
R_{\phi \phi} & =-\frac{\left(4 r \frac{\partial^{2} r}{\partial v \partial u}-h+4 \frac{\partial r}{\partial v} \frac{\partial r}{\partial u}\right) \sin ^{2} \theta}{h}
\end{aligned}
$$


Setting $\Omega=u$, and again using (28), the conformally related Ricci tensor has nonzero components

$$
\begin{aligned}
& \widetilde{R}_{u u}=\frac{2\left(-u h \frac{\partial^{2} r}{\partial u^{2}}-r \frac{\partial h}{\partial u}+u \frac{\partial h}{\partial u} \frac{\partial r}{\partial u}\right)}{u h r} \\
& \widetilde{R}_{u v}=\frac{u r \frac{\partial h}{\partial u} \frac{\partial h}{\partial v}-u h r \frac{\partial^{2} h}{\partial v \partial u}+2 h^{2} \frac{\partial r}{\partial v}-2 u h^{2} \frac{\partial^{2} r}{\partial v \partial u}}{u h^{2} r} \\
& \widetilde{R}_{v v}=-\frac{2\left(h \frac{\partial^{2} r}{\partial v^{2}}-\frac{\partial h}{\partial v} \frac{\partial r}{\partial v}\right)}{h r} \\
& \widetilde{R}_{\theta \theta}=-\frac{4 u r \frac{\partial^{2} r}{\partial v \partial u}-u h+4 u \frac{\partial r}{\partial v} \frac{\partial r}{\partial u}-8 r \frac{\partial r}{\partial v}}{u h} \\
& \widetilde{R}_{\phi \phi}=-\frac{\left(4 u r \frac{\partial^{2} r}{\partial v \partial u}-u h+4 u \frac{\partial r}{\partial v} \frac{\partial r}{\partial u}-8 r \frac{\partial r}{\partial v}\right) \sin ^{2} \theta}{u h}
\end{aligned}
$$

Computing the pullback of $\Omega\left(R_{a b}-\widetilde{R}_{a b}\right)=u\left(R_{a b}-\widetilde{R}_{a b}\right)$, the relevant components are

$$
\begin{aligned}
& u\left(R_{v v}-\widetilde{R}_{v v}\right) \longrightarrow 0 \\
& u\left(R_{\theta \theta}-\widetilde{R}_{\theta \theta}\right) \longrightarrow \frac{-8 r \frac{\partial r}{\partial v}}{h}=\left(\frac{-8 \frac{\partial r}{\partial v}}{r h}\right) r^{2} \\
& u\left(R_{\phi \phi}-\widetilde{R}_{\phi \phi}\right) \longrightarrow \frac{-8 r \frac{\partial r}{\partial v}}{h} \sin ^{2} \theta=\left(\frac{-8 \frac{\partial r}{\partial v}}{r h}\right) r^{2} \sin ^{2} \theta
\end{aligned}
$$

so that

$$
\underset{\Omega\left(R_{a b}-\widetilde{R}_{a b}\right)}{\longleftarrow}=\left(\frac{-8 \frac{\partial r}{\partial v}}{r(0, v) h(0, v)}\right) q_{a b}
$$

Thus the conditions of Theorem 4 are satisfied. More importantly, the conformal transformation $\bar{g}_{a b}=\left(\omega^{2}\right) g_{a b}$ with $\omega=r(u, v)$ will yield $£ \bar{n}_{a b}=0$, the condition needed to use the pullback to produce a well-defined covariant derivative on $\Sigma=\{u=0\}$.

Again, straightforward computation verifies that the pullback connection constructed from the conformally related metric is indeed well-defined on $\Sigma$.

\section{Summary}

Due to the degenerate metric, working with null surfaces offers some very challenging obstacles, since traditional tools such as Christoffel symbols are not defined. Gauss decomposition fails, since there are non-zero null vectors both tangent and perpendicular to the hypersurface. The work of Duggal and Benjacu attempts to overcome this difficulty by defining a screen manifold and a lightlike transversal vector bundle to decompose the manifold and the null hypersurface. However, even with all of this structure, there are elementary examples, 
such as the null cone, that do not satisfy the hypotheses necessary to produce a covariant derivative independent of the screen.

An alternate construction uses the pullback to define the covariant derivative. Care must be taken when using this technique, since the resulting derivative operator may not be well defined; this technique will only work as long as the null vector field is a Killing vector field.

Motivated by the work of Geroch on asymptotically flat spacetimes, conformal transformations were used not only to give a well-defined derivative on null hypersurfaces, but also to provide a test to determine whether the null surface admits such a definition.

Finally, the conformal pullback method was shown to work at the horizon of the Schwarzschild geometry, and more generally for any spherically symmetric spacetime.

Further work is needed to understand the implications of this construction. For example, in the case of the null cone, the conformal transformation results in a null cylinder. What does it mean to use the cone's covariant derivative operator on a sphere? And are there non-symmetric null surfaces on which this construction works?

Finally, we remark that there does not appear to be a similar technique for Riemannian spaces, but since traditional Gauss decomposition works, this technique is not needed in that case.

\section{Acknowledgments}

This paper is based on work submitted by DH in partial fulfillment of the degree requirements for his Ph.D. in Mathematics at Oregon State University [1].

\section{References}

[1] Don Hickethier Covariant Derivatives on Null Submanifolds, PhD Thesis, Oregon State University, December 2010. (Available online at: http://hdl.handle.net/1957/19547.)

[2] Robert Geroch, Asymptotic Structure of Space-Time, in: Asymptotic Structure of SpaceTime, F.P. Esposito and L Witten, eds., Plenum Press, New York and London, 1976, pp. $1-105$.

[3] Krishna L. Duggal \& Aurel Bejancu, Lightlike Submanifolds of Semi-Riemannian Manifolds and Applications, Kluwer Academic Publishers, Dordrecht/Boston/London 1996.

[4] Michael Spivak, A Comprehensive Introduction to Differential Geometry, 2nd edition, vol. 3, Publish or Perish, Houston 1979. 